\title{
Synchronizing programs using Macromedia Director plug-ins: An example from the field of developmental psychopathology
}

\author{
W. LOCKE MORGAN and CARL F. WEEMS \\ University of New Orleans, New Orleans, Louisiana
}

\begin{abstract}
Synchronizing the display of a stimulus with physiological recording or subjects' behavioral responses is crucial for acquiring valid measures of performance. This article describes a technique for integrating the presentation of stimuli, behavior reports, and physiological data employing Macromedia Director and a standard biometric program using an example from the field of developmental psychopathology. Our experience has been that the use of this technique is effective in creating complex protocols for research. Although the temporal accuracy available from this particular method is adequate for some experiments, it is not suited for investigations requiring single millisecond accuracy. Considerations and suggestions for utilizing this and similar techniques for integrating data collection and stimuli presentation are discussed.
\end{abstract}

Psychological experiments often require the simultaneous presentation of a stimulus and the collection of data. For example, a researcher may record behavioral, physiological, or cognitive responses timed to the presentation of various stimuli. The field of psychopathology provides specific examples (see, e.g., Sawchuk, Lohr, Westendorf, Meunier, \& Tolin, 2002; Thorpe \& Salkovskis, 2000). Sawchuk et al. collected data from participants with spider phobia $(n=39)$, and with general phobias $(n=37)$, as well as from participants who were nonphobic $(n=40)$. Participants viewed fear-provoking and disgusting pictures displayed on a computer screen and video from a VCR displayed on a TV, and they reported their responses to these stimuli with paper and pencil questionnaires. In a study on memory bias concerning spider information, Thorpe and Salkovskis collected data from participants with spider phobia $(n=31)$ and nonspider phobias $(n=$ $20)$ as well as from nonphobic individuals $(n=15)$, using a TV/VCR combination to present a video of spiders and paper questionnaires to record responses. The equipment used is not unusual for conducting these types of experiments, but it lacks the advantages offered by computerized integration. In this article, we describe a technique to integrate the presentation of stimuli, behavior self-reports,

This research was supported in part by a grant from the National Institute of Mental Health (MH067572) awarded to C.F.W. Correspondence concerning this article, including requests for copies of the code, should be addressed to Carl F. Weems, Department of Psychology, University of New Orleans, New Orleans, LA 70148 (e-mail: cweems@uno.edu).

Note-This article was accepted by the previous editor, Jonathan Vaughan. ,and physiological data using Macromedia Director with a standard biometric program using an example from the field of developmental psychopathology.

The lack of connection between the components in the studies above decreases the efficiency of the procedure and leaves many opportunities for experimenter error. Using the integration techniques we describe here, the presentation of a stimulus and the recording aspects can be closely synchronized so that the researcher is better able to precisely compare the data collected at pre- and poststimulus presentations. Other benefits may include the recording of responses outside of the program that collected the responses; automatic or timed placement of markers in biometric waveforms when the initiation of the marker is prompted by events in another program, such as a program that presents stimuli; and the ability to increase protocol flexibility by setting the pace to the participant (e.g., video could be sectioned into discrete clips that can be run or held in waiting as the situation demands).

The technique that will be described here was developed for the presentation of multimodal stimuli (audio and visual), collection of memory responses, and collection of physiological data. Briefly, the example we use in this article is an integrated multimedia program for testing cognitive and biological theories of childhood anxiety disorders (e.g., Weems, 1999; Weems, Berman, Silverman, \& Saavedra, 2001). It was constructed by uniting multimedia software with biofeedback software and hardware. The multimedia software used was Macromedia Director (www.macromedia.com), and the biofeedback software was BioGraph accompanied by its hardware counterpart, the ProComp + (Meyers, 1999). Director is a multimedia development program, which was used to create a self-contained program, referred to as a "projector." Below we outline the development of the program and its functions. 


\section{METHOD}

\section{Measures and Program Development}

The program in our example was designed on the PC platform (Microsoft Windows 2000) to present a participant with video stimuli, collect self-report data (primarily memory data and self ratings of fear), collect biometric data (heart rate and skin conductance), automatically place markers in the biometric data, and output the results to both a computer file and a printer. Most of this was done through Macromedia Director's development environment for the purpose of creating a self-contained program, known as a projector. The environment is run by a Java-like (Schildt, 2003) script known as Lingo (Rosenzweig, 2001), which controls a timeline layout that is composed of channels (e.g., video and audio) and frames (e.g., motion pictures). Once the projector is designed, it can be run on the average Windows system. Copies of the code used in the example presented here are available from the authors upon request. The program was developed and run on a Dell OptiPlex GX400, Pentium 4, 2.4GHz, 128MB RAM computer, with dual monitors running on a Radeon 7000 AGP video card. Minimum requirements are a Pentium 3,350MHz, 128MB RAM CPU, a graphics card that can run two monitors, and sound capability to run the example presented here.

For this particular project, the video was digitized using Pinnacle Systems Dazzle software (www.pinnaclesys.com) and saved into clips as MPEG1 files. These clips were then placed into the timeline of the Director environment at regular intervals, and an initial interface (i.e., the first interactive frame of the program) was constructed. This interface allowed the participant to enter his/her identification number and the test condition to which the individual had been assigned. Again using Macromedia Director's Lingo, buttons were created to provide the researcher with an aid for introducing the participant to the protocol. Each button was programmed to prompt events, such as a demonstration of the stimuli to be expected, starting a timer, and beginning the procedure itself. During the time that the interface is displayed, a master script (or in Director, a "movie" script) is run that sets all the initial variables and seeks the BioGraph program for later referencing. This last function establishes interconnectivity among the programs.

Interconnectivity allows automatic placing of markers in the biometric feedback waveforms. The BioGraph program was designed to lay down markers when manually instructed to do so by the operator. Our task was to automate this feature of BioGraph to the presentation of each stimulus. At present, this is not a feature that Director can accomplish on its own, but one that requires a plug-in or plug-ins (or Xtras, as Director calls them). We used the Buddy API v3.6 (by Magic Modules, www.mods .com.au) and the MasterApp v1.06 (by UpdateStage, www.updatestage.com) Xtras. When the interface button for beginning the protocol is pressed, a script is run that checks whether all the appropriate fields are filled in, then records the information to a list. It also records the time, in milliseconds, that the program has been running since the "Begin Record" button was pressed. This serves as a fairly precise reference, but does not imply millisecond accuracy, and initiates interaction with the BioGraph program for the purpose of laying down a marker. After identifying the main window of the BioGraph program, the Director plug-in brings it to the front and sends a simulated $<$ return $>$ keystroke to it. BioGraph then opens a dialog box into which the plug-in types a label and closes the dialog by sending another simulated $<$ return $>$ keystroke. Dual monitors allow the projector to be on one screen and BioGraph to be on the other, therefore the switch is not apparent to the participant. The final result is that, in the time required to cycle the internal message loop of Windows, BioGraph has inserted a marker into the biometric data in an objective and systematic way. The temporal accuracy of the marker is determined by the time required to execute the process (i.e., the exact timing or precision of the marker placement is a function of the processing). Although our research protocols do not require millisecond accuracy, additional estimates of timing accuracy can be completed as needed. A suggestion for such testing is described below. What is known is that BioGraph places the marker at the reception of the first $<$ return $>$ key and then adds the label. Therefore, with six messaging loops being run prior to the first $<$ return $>$ key, the accuracy is dependent on the speed at which these tasks are processed. This dependency on task processing does point to a limitation of the methodology in that the accuracy is not well suited for millisecond-critical recording. This is addressed further in the Discussion section.

The Buddy API and the MasterApp Xtras accomplish interconnectivity together by accessing the underlying API of Windows (including Windows 98, 2000, and NT). The programmer is not required to understand the API of Windows as the Xtras serve as "black boxes." The programmer simply needs to call the desired methods in the API, and the action is performed. For example, the method for freeing up the processor from the demands of the projector by temporarily pausing its code execution is "mappFeedGenericTimeSlice." (Any method that resides in MasterApp begins with "mapp," and any method that resides in Buddy API begins with "ba.") Descriptions of how to employ the methods are on the relevant Web sites (see above). Both Buddy API and MasterApp are designed by solid companies that support their products and plan future versions.

Director can display AVI and Quicktime (www.apple.com/ quicktime) video without the assistance of Xtras, but cannot yet support MPEG formats. MpegXtra (by Tabuleiro, xtras.tabuleiro.com) is an Xtra that allows MPEG video to be run in a Director presentation. Director, through MpegXtra, treats an MPEG file similarly to how it would run AVIs. The programmer can define such things as the frame to start from, the frame to end on, and the rate at which the video is played. In our research, no cognitive response data were collected during the playing of video, nor were markers placed (i.e., they came before and after the video, and thus there was no interconnectivity attempted 
during the video). Therefore, we have not assessed timing issues that may arise during the processor-heavy task of playing video. However, the operation of BioGraph and collection of physiological response did not seem to be impaired by the playing of video (see below).

In the final steps of the program, two other functions were activated to write the responses to a file and output the same to a printer. The file writing was done through the FileIO Xtra that is included with Director. This Xtra allows Director to perform simple functions like reading and writing on text documents. In this case, the projector was programmed to extract the accumulated data from a list and to seek a text document by name and location relative to the main projector file. If the document is present, then the data is appended to the file, otherwise a new file is created and the data written to it.

A similar operation was used in the printing function. The Print-o-Matic Xtra (by Electronic Ink, www.printomatic.com) harnesses the data, which includes a screenshot of one of the measures, and creates a print file. While the data are taken from the same list variable that was used in writing to the file, "capturing the stage" makes the screenshot ("stage" refers to the area of activity that is presented to the participant). This is done by prefabricating an "empty" graphic file that is the same resolution as the intended screenshot, capturing the image, and replacing the empty graphic's image with the newly acquired one. This image is then added to the print document, and a standard Windows print dialog is displayed.

\section{The Study Protocol}

The goal of the protocol was to assess cognitive and physiological responses to a mild phobic stimulus (i.e., video of a German shepherd running toward the screen). The biometric device was attached to the fingers of the person as done in previous research and as recommended by the manufacturer (e.g., Meyers, 1999; Weems, 1998). Once all the receptors were attached, BioGraph and ProComp + were started and turned on, respectively, and the biometric signals were shown in BioGraph. The experimenter pressing the "start timer" button captured the timing in milliseconds from the computer's internal clock and enabled the projector to keep track of the milliseconds that elapsed between the various portions of the protocol. As noted, this does not imply that millisecond accuracy was achieved, but this method does serve as a fairly precise reference point in the data. For our purpose, which was to examine changes in pre- and postvideo HR and GSR response, the accuracy was very satisfactory. Next, the "begin test" button was pressed and the projector displayed a blank gray screen for $10 \mathrm{sec}$. This allowed the biometric recording to establish a baseline. From this point, the protocol moved into a series of measures and stimuli presentation (e.g., video of phobic and nonphobic scenes, fear and memory rating measures). At the end, the experimenter was prompted to print the results and instructed to stop the biometric recording, save those results, and remove the sensors from the fingers of the participant.

\section{ILLUSTRATIVE OUTCOME AND DISCUSSION}

In our experience with the technique, all of the test participants completed the experimental procedure. The data from each test were appropriately saved in a text file, which was, in turn, converted to SPSS format. The correct markers were placed in the biometric recording, and the printout of the self-reported responses was properly generated. SPSS accepted the 112 variables, and the printouts that were automatically generated at the end of the protocol were consistent with the expected appearance. The list of the collected data is a reassuring physical copy of the data.

The biometric output can be reviewed in BioGraph as the entire waveform, in a condensed form as a printout, or as raw data in a spreadsheet. The printout method allows for quick appraisal of the session. The spreadsheet form of the biometric data places values that were sampled from the incoming signal into individual cells of a spreadsheet so that they can be used for data analysis. Heart rate (beats per minute) and amplitude (beat amplitude percentage) were sampled at $32 \mathrm{~Hz}$, and GSR and raw pulse (EKG) were done at $256 \mathrm{~Hz}$. The "export" feature of BioGraph made the transition from waveforms to numbers a fairly simple task. Sections for export were defined based on the markers that had been dropped into the biometric data stream (e.g., so that pre- and poststimulus presentation could be compared).

Although the ProComp + appliance and BioGraph are widely used in clinical settings and are commonly used in tandem with multimedia displays (Klein, 2002) to ensure the accuracy of sampling, we inspected the resulting waveforms of the biometric data and the data output (in SPSS) in a random sample of 20 participants from our research studies. Visual inspection of the waveforms suggested that the accuracy is maintained, even when video is presented, because of the consistent and continuous appearance of the waveforms. If there had been a problem, there would have been simultaneous "flat lines" across all the channels not due to the biometric device slipping off, which typically affects either GSR channels or HR channels, but not both (i.e., it is unlikely that both devices would become detached). Regarding the accuracy of the program, the data files show no more artifacts (HR responses less than 50 beats per min or greater than 170 are eliminated because they are highly improbable responses in this age group) or missing data in the more complicated segments (e.g., while video is running) than in the lowdemand segments (e.g., baseline). We calculated for example a "valid HR data score" as the number of valid (i.e., nonmissing, nonartifact) HR data points divided by the number of data points possible for the baseline segment and for the first video segment. Using a paired sample $t$ test, we found no significant differences between segments [mean difference $=-.052 ; S E$ of the mean $=.051$; $t(19)=-1.01, p=.32]$.

There are additional methods that researchers may use to test timing accuracy and to verify the accuracy of data 
collection when using protocols developed with the technique described in this article. For example, temporal accuracy of the marker placement can be further verified by utilizing a copy of the code for the final protocol that includes several immediate and successive placements of markers. Writing the code to place the markers as close together as possible will allow the researcher to examine the time between any two markers, and this time will represent the time required to place a single marker in their program. To verify that the program has not interfered with data collection, researchers may wish to record a known waveform on a spare channel so that any missed sampling would be obvious. The developers of BioGraph used this technique to test their product; however, researchers interested in further testing their data streams, or those using other biometric recording software, may wish to employ this in their study protocol. This can be accomplished by using a voltage isolator in conjunction with the ProComp + (or other biometric device). If the voltage isolator is set to emit a sine wave pattern, for example, then visual inspection of the resulting output should give contrast to any flaw in the recording. Problems such as an overburdened processor, inadequate memory, and program interference should become readily apparent in any incorrect tangent of the sine wave pattern.

Director is not the least expensive means for program development. Other avenues exist for the able programmer/psychologist. For instance, one could build a program using programming languages such as Java, Visual Basic, or $\mathrm{C}++$. These are "object oriented" languages that are relatively simple compared to a "low level" language like Cobol, which would likely require a computer science degree (or a lot of hobby time) before one could produce a program (Ruknet, 1995). It is our opinion that Director is an example of a program that is adequate for presenting stimuli for a variety of applications in developmental psychopathology research but that may not be useful for research on reaction time or psychophysiology research examining just noticeable differences that require millisecond resolution. Thus, the limitation of the present software configuration is its lack of resolution for tests demanding very high precision (see, e.g., Schneider, Zuccolotto, \& Tirone, 1993). This observation does not imply that BioGraph, in and of itself, cannot maintain millisecond resolution.

Although Director has the ability to communicate with external programs and devices through Xtras, it does not have the level of priority with the major Windows operating systems that would be required for tests such as a signal detection threshold task (Brysbaert, 1994). This may also be an attribute of other operating systems, but our experience has not verified this. Director employs
Windows's internal message loop and event queue, which are not reliable to millisecond accuracy. At present, the overlay of multimedia components within Director make the resulting output susceptible to yielding processing time to other tasks. This means that it will simply skip, or hold over, a frame of functioning if some other program requires the attention of the processor. Naturally, for most situations, a programmer can limit the burden on the processor and achieve a flawless presentation, but the more intensive the program becomes, the more care must be taken to ensure accuracy. One specific innovation that is available is the "mappFeedGenericTimeSlice" method in the MasterApp Xtra. This puts a hold on the projector's processor time and allows other tasks to process. We employed this method in our integration technique, even though the current application does not require a millisecond-bymillisecond level of precision. Other standard practices, such as avoiding screen redraw, unnecessary variable checking, or unnecessary high-resolution graphics, may also be used to ensure accuracy.

\section{REFERENCES}

BRYSBAERT, M. (1994). Behavioral estimates of interhemispheric transmission time and the signal detection method: A reappraisal. Perception \& Psychophysics, 56, 479-490.

KLEIN, L. (2002). Where is biofeedback heading? Biofeedback, 30, 1921.

Meyers, H. (1999). ProComp+/BioGraph Biofeedback System (Version 2.1) [Computer Program]. Montreal, PQ: Thought Technology Ltd.

RosenzweIG, G. (2001). Special edition: Using Macromedia Director 8.5. Berkeley, CA: Pearson Education.

RuKNET, C. (1995). A guide to programming languages: Overview and comparison. Boston: Artech House.

Sawchuk, C. N., Lohr, J. M., Westendorf, D. H., Meunier, S. A., \& Tolin, D. F. (2002). Emotional responding to fearful and disgusting stimuli in specific phobics. Behaviour Research \& Therapy, 40, 1031-1046.

ScHILDT, H. (2003). Java 2: A beginner's guide. New York: McGraw-Hill.

Schneider, W., Zuccolotto, A., \& Tirone, S. T. (1993). Time-stamping computer events to report .1-msec accuracy of events in the Micro Experimental Laboratory. Behavior Research Methods, Instruments, \& Computers, 25, 276-280.

Thorpe, S. J., \& SAlkovskis, P. M. (2000). Recall and recognition memory for spider information. Journal of Anxiety Disorders, 14, 359-375.

WeEMS, C. F. (1998). The evaluation of heart rate biofeedback using a multi-element design. Journal of Behavior Therapy \& Experimental Psychiatry, 29, 157-162.

WeEMs, C. F. (1999). Conceptual and methodological issues in examining behavioral development through environment-organism interactions. New Ideas in Psychology, 17, 149-164.

Weems, C. F., Berman, S. L., Silverman, W. K., \& Saavedra, L. S. (2001). Cognitive errors in youth with anxiety disorders: The linkages between negative cognitive errors and anxious symptoms. Cognitive Therapy \& Research, 25, 559-575.

(Manuscript received February 6, 2004; revision accepted for publication August 21, 2004.) 